\title{
The Design of Intelligent Charger for Solar energy Based on SCM SHE Yan
}

\author{
Shunde Polytechnic, Foshan 528333, China
}

Shelley186@sohu.com

Key Words:Solar energy; intelligent charge; CUK converter; SCT12C5A60S2

Abstract:The design of intelligent solar charging system, through the solar panel to convert solar energy into electrical energy, through DC/DC converter circuit processing, charging the battery. The entire system consists of DC/DC conversion circuit, a processor module, A/D sampling circuit, pulse width modulation controller, a display circuit and a battery group. The process of charging the battery is controlled by pulse width modulation, thereby output power of the solar cell and battery life are improved, extend the battery service life.

\section{Introduction}

Due to the increasing depletion of fossil fuels, people emphasis on environmental issues is also rising, looking for clean alternative energy is becoming increasingly urgent. Solar energy as a renewable energy it has inexhaustible and clean safety features, has broad application prospects. When using the solar cell sunlight due to the large changes in the internal resistance and relatively high, so the output voltage is unstable, the output current is small, which need to be supplied with a charge control circuit converts the DC voltage regulator after the solar panels output battery. When the light conditions are suitable to absorb sunlight through solar panels, convert light energy into electrical energy. Since the use of high-current charger quick charge method, the battery is full, if not stopped in time cause the battery hot, excessive charging will seriously damage the battery life, which requires a complex control system. The system uses STC12C5A60S2 MCU as controller charging circuit so as to lower the cost easily implement complex intelligent charge control.

\section{Overall System Design}

The overall design of the system is shown in figure 1, through solar panels convert solar energy into electrical energy, achieved by the microcontroller programming PWM wave control switch in order to achieve the output voltage and current's, change the output state is displayed by the display circuit and sizes, from the STC12C5A60S2 with the AD converter to achieve acquisition and conversion data, and make a judgment process, making intelligent output and control circuit[1][2]. The system consists of DC/DC converter circuit, the processor module, A/D sampling circuit, PWM pulse width modulation controller, display circuit and battery components.

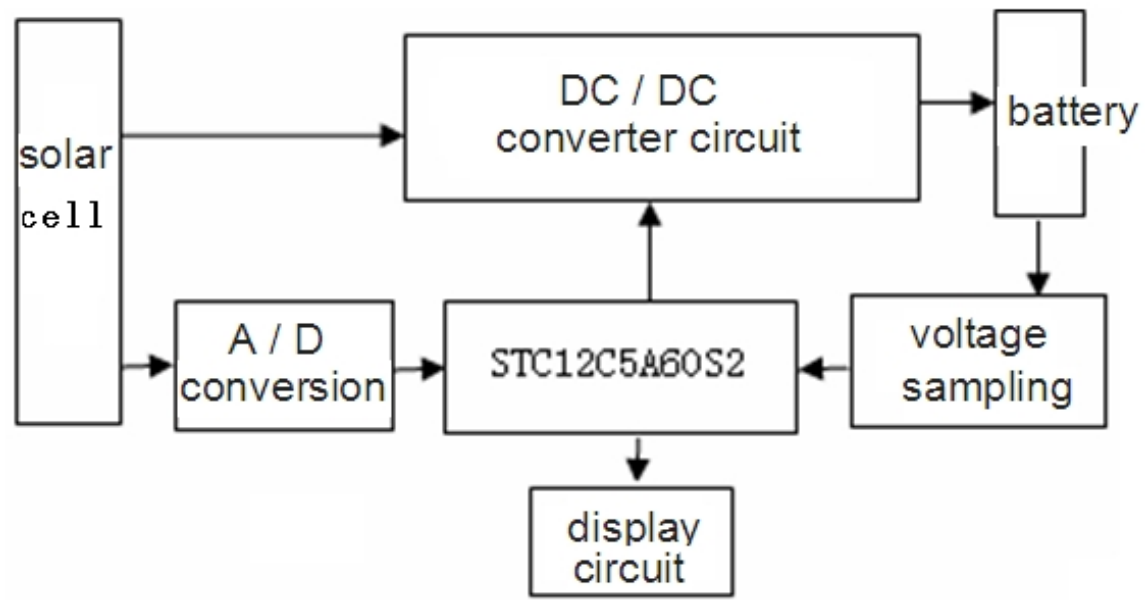

Fig.1 the overall design diagram 


\section{The Main Circuit DC/ DC Converter cCircuit}

DC / DC converter circuit is to rely on the switching operation of the semiconductor switching devices, the DC voltage is converted into a DC voltage to another circuit. Cook (Cuk) conversion circuit belongs to the buck-boost DC / DC converter circuit shown in Figure 2. The figure for the inductor L1 and L2, D is the fast recovery freewheeling diode, C1 is transferring energy coupling capacitor, C2 filter capacitor. Characteristic of this circuit is that the output voltage to the input voltage polarity is reversed, the input terminal of the current ripple, output DC voltage stable, reducing external filtering requirements.

Output voltage and input voltage relationship of the circuitry is $U_{0}=-\frac{D}{1-D} U_{d}$. Minus sign indicates the output voltage and the input voltage is inverted. To output a certain voltage U0 value, must be given the duty ratio $\mathrm{D}$ corresponding value depending on the value of the supply voltage Ud of sampling[3].

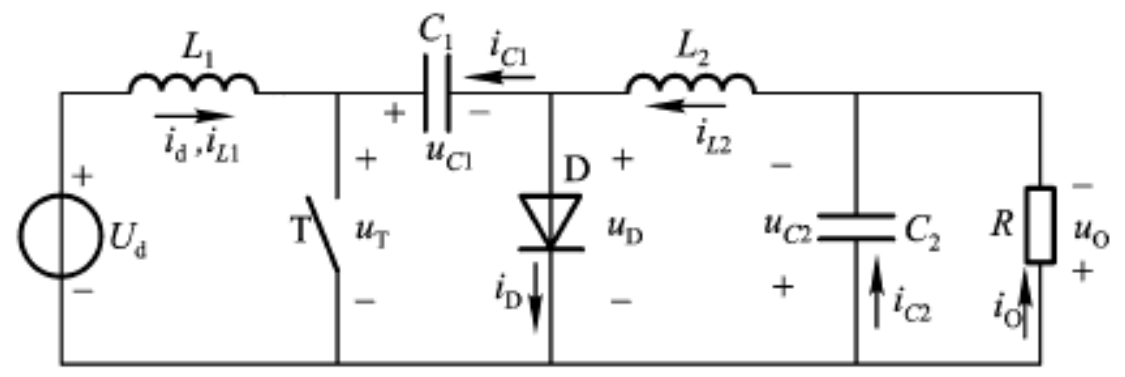

Fig.2 the CUK circuit diagram

\section{The Main Processing Module}

This design uses STC12C5A60S2 as the main processing chip. STC12C5A60S2 / AD / PWM MCU is a macro crystal technology production of single clock / machine cycle (1T) of the microcontroller, high-speed / low power / super anti-jamming new generation of 8051, the instruction code is fully compatible with the traditional 8051, but the speed 8-12 times faster. Dedicated internal integration MAX810 reset circuit, 2 PWM, 8-way high-speed 10 A / D converter (250K / S, or 25 million times / sec), for motor control, strong interference occasions.

ADC Sampling Circuit. STC12C5A60S2 MCU ADC is a successive approximation type ADC. Successive approximation type ADC consists of a comparator and D / A converter constituted by successive approximation logic, from the most significant bit (MSB) starts sequentially for each of the input voltage and the built-in D / A converter output compare, after several comparison, so the resulting digital converter successive approximation analog input corresponding value. Successive approximation type A / D conversion rate of up to 250K / S (per 250,000). 10 precision $\mathrm{ADC}$, a total of eight.

MCU in the control variable duty cycle PWM wave output, with solar panel output voltage to match the original, is determined by the formula $|D|=\frac{U o}{U o+U d}$. Therefore, the controller needs to determine the original value Ud, which must be A / D sampling. As the device working voltage of $5 \mathrm{~V}$, and output from the solar panel voltage $0-20 \mathrm{~V}$, it may exceed the operating voltage and burned chip, so before the A / D sampling, the need for accurate blood pressure to sampling[1][2]. The design $\mathrm{Uo}=1 / 4 \mathrm{Ud}$. Proportional arithmetic circuit is as shown in figure 3. 


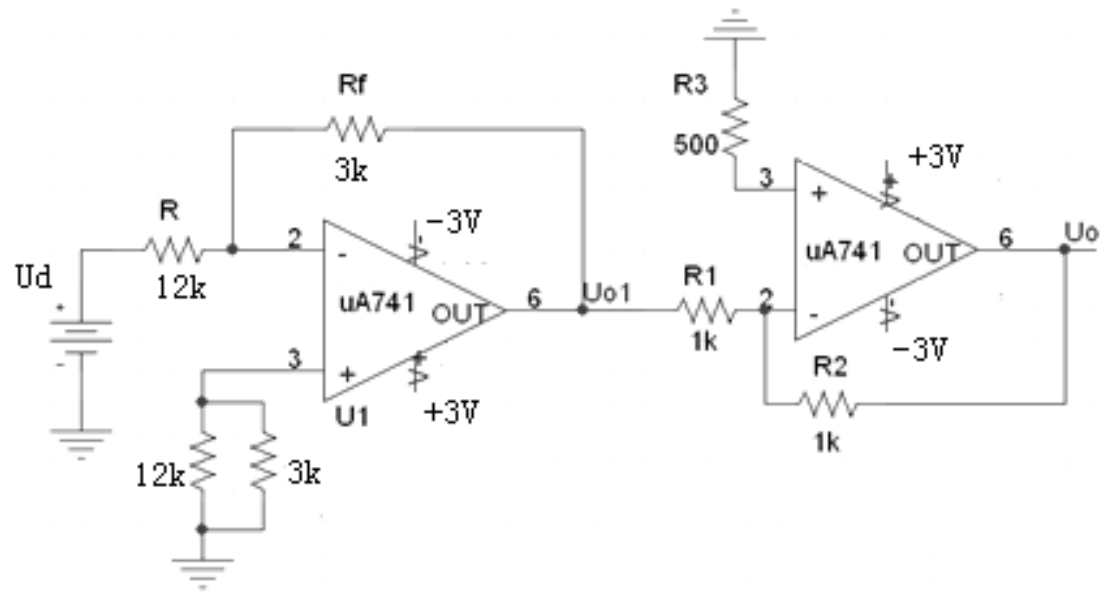

Fig.3 the proportional amplifier sampling circuit diagram

Buck ratio arithmetic circuit can be accurately obtained by the single-chip sampling results of the exact value of the original solar panel output voltage, thereby controlling the duty cycle of the output voltage to match the original PWM wave.

Display Circuit Design. LCD display with its micro-power consumption, small size, display rich content, ultra-thin lightweight many advantages, more and more widely used in the pocket instrumentation and low-power applications. Character LCD module is a dedicated display symbols dot matrix LCD, according to the capacity of the display can be divided into one line of 16 characters, 2 lines of 16 characters, 2 lines of 20 characters, etc., the design uses two rows * character LCD1602 LCD display with backlight 16, showing the charging process and sunlight intensity[4]. LCD wiring diagram shown in figure 4.

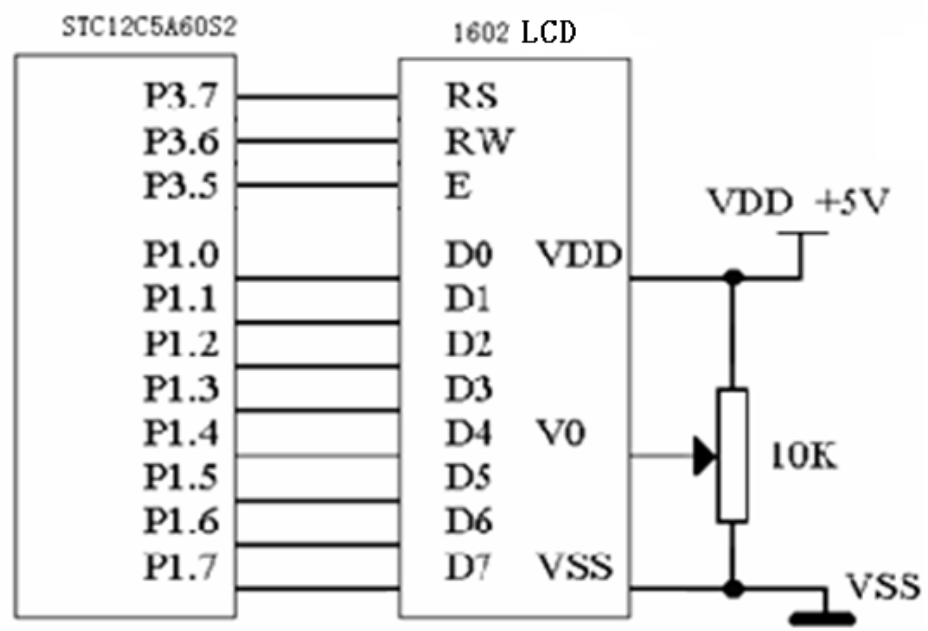

Fig.4 the LCD1062 wiring diagram

\section{System Software Design}

The SCM system is the main task of process control data acquisition, and data collected after the analysis process generates a PWM signal controlling the switch is turned on and off to control the output size. Specific work process is power-on reset, first detecting the intensity of sunlight whether the charging requirements, reached the decision after charging by comparing the detected voltage and current signals, and then transferred to the appropriate routine analysis to calculate the PWM duty cycle, the output of current or voltage, and the display data to the display circuit. In the course of the microcontroller output timer detects the output current or voltage, compare with the set value adjusted PWM duty cycle, the output tends to set value. In the battery charging process, the current size is determined by detecting how much charge the battery, thereby changing the charging mode or decide whether to stop charging[5][6][7].

Circuit Starts Initialization. Initialization is to set the initial operating environment for the MCU operation, mainly to complete the following tasks: internal clear sheet, initial parameter 
settings, AD set, set the timer and interrupt settings.

Display Routines. Display program progress display for displaying the charging process, as from the start to enter the charging system and the end of the interactive interface, the use of the LCD1602 LCD module, it can only display letters, numbers and special symbols. Including whether the display intensity of sunlight charging requirements, the charging process and the end of charging in English.

Data Acquisition and Analog to Digital Conversion Process. Data acquisition and analog-digital conversion data initialization procedure completed, transmit start conversion command, wait for the end of conversion, data is received, processed and stored in the cache.

Charge Subroutine Design. The charging process is divided into two phases, the first phase of a constant current, the charging current can be set into the second stage when the charging voltage reaches $4 \mathrm{~V}, 4.2 \mathrm{~V}$ constant voltage charging mode ie, the constant charging current over time goes gradually decreased until the charging current is reduced to $0.1 \mathrm{~mA}$, indicating that the battery charge will gradually be reduced to zero, the battery is fully charged. During charging, the LCD display "charging"; when full, the LCD screen display "Charge completed", if not enough light intensity is displayed "Sun intensity insufficient".

\section{Conclusion}

Design of the solar charging system is divided into hardware design and software design in two parts, the hardware circuit is completed by the STC12C5A60S2 MCU control and design of the main circuit and the determination circuit schematic design and parameters. Software design is modular programming method, it is divided into main section, AD converter and PWM pulse acquisition module signal generation module. According charger experimental sample design made by setting parameters and microcontroller programming, were tested. Experimental results show that the charger is the good performance, in line with the design requirements, has a simple structure, low cost and high reliability.

\section{References}

[1] STC12C5A60S2 MCU Device Handbook [EB / OL].

[2] Li Xiuzhong. Principles and Applications [M]. South China University Press, 2009.8.

[3] Huan Ximing, Yao Weizheng. Power Electronics Technology [M]. Higher Education Press, 2010.9 .

[4] LCD LCD1602 Manual [EB / OL].

[5] Jiang Hongfei, Hu Shuting Green Energy - Solar Charger [J], Shanghai Institute of Technology, 2007.2.

[6] Liu Chao Smart Phone Battery Charging System Based on Microcontroller [J], Changchun University of Technology (Higher Education Edition), 2007.1.

[7]Chen Jie.Design and Research of Solar multifunction charger [J]. Electronic Technology, 2008.8. 